\section{Mindfulness-Based Cognitive Therapy Associated with De- creases in C-Reactive Protein in Major Depressive Disorder: A Pilot Study}

\section{Stuart J Eisendrath*, Erin Gillung, Abby Hartzler, Molly James-Myers, Owen Wolkowitz, Walter Sipe, Dhakshin Ra- manatham and Kevin Delucchi}

Department of Psychiatry, University of California, San Francisco, California, USA

\begin{abstract}
There appears to be growing evidence that there is a relationship between major depressive disorder and inflammation. This study investigates the relationship between depression and C-Reactive Protein (CRP), a marker for inflammation. We utilize mono-treatment with Mindfulness-Based Cognitive Therapy (MBCT) as the independent variable in this investigation. To our knowledge, this is the first study to explore whether MBCT mono-treatment may affect an inflammatory biomarker in major depressive disorder. In an open trial, 11 participants had blood samples taken pre- and post-intervention. Results indicate that there significant decreases in CRP levels as well as depression severity ratings. These results suggest that further investigation with a randomized controlled trial could yield more substantial information about the relationship of inflammation and the psychotherapeutic treatment of major depression. This information may have implications for future targets of depression treatment.

Trial registration: CurrentTrials.gov Identifier; NCT02385786 Registered 10 March 2015.

Keywords: C-Reactive protein; Inflammation; Major depressive disorder, Meditation; Mindfulness; Mindfulness-based cognitive therapy; Psychotherapy
\end{abstract}

\section{Abbreviations}

CRP

HAMD-17

MDD

MBCT
C-Reactive Protein

Hamilton depression rating score-17 Major Depressive Disorder Mindfulness-Based Cognitive Therapy
*Corresponding author: Stuart Eisendrath, Department of Psychiatry, University of California San Francisco, 401 Parnassus Avenue, San Francisco, CA 94143-0984, USA, Tel: +1 4154767868; E-mail: stuart.eisendrath@ucsf.edu

Citation: Eisendrath SJ, Gillung E, Hartzler A, James-Myers M, Wolkowitz O et al. (2016) Mindfulness-Based Cognitive Therapy Associated with Decreases in C-Reactive Protein in Major Depressive Disorder: A Pilot Study. J Altern Complement Integr Med 2: 010.

Received: April 14, 2016; Accepted: June 02, 2016; Published: June 16, 2016
QIDS-SR $_{16}$

RCT

Quick Inventory of Depressive Symptoms-Self Report-16

\section{Background}

The role of inflammation in depression is being investigated in several directions. While it remains unclear whether inflammation precedes or is a consequence of the depressive state, many studies have shown increased inflammatory biomarkers in depressed patients compared to healthy controls [1-4]. We examined the effect of a psychotherapeutic intervention, Mindfulness-Based Cognitive Therapy (MBCT), on the acute phase protein C-Reactive Protein (CRP), a reliable biomarker of increased inflammation [2,5], in depressed patients without medical illness. While some studies have shown inflammatory biomarkers to return to normal after antidepressant treatment [6,7], and inflammatory markers to decrease in medically ill patients who underwent psychotherapeutic intervention [8,9], our preliminary investigation is the first to our knowledge that explores whether MBCT mono-treatment for Major Depressive Disorder (MDD) in individuals without medical illness may affect an inflammatory biomarker as well as mood.

The relationship between depression and inflammation is still evolving. Harley et al., [10] noted that elevated CRP is a predictor of good long-term outcomes with antidepressant treatment and poor outcome with psychotherapy, although there was only a small sample of individuals with high CRP. Dahl et al., [11] investigated cytokines and CRP in depressed individuals receiving nonpharmacological treatment with weekly support and psychotherapy as compared with those receiving antidepressants. Both samples had reductions in cytokines with recovery, the nonpharmacological sample significantly so. Neither sample had changes in CRP. Krogh et al., [12] investigated the effects of exercise on IL-6 and CRP in a depressed population. They noted that there were no significant changes in either parameter after 3 months. Clearly there is further explorations are needed.

\section{Methods}

This study was an open trial of MBCT as a mono-treatment for major depressive disorder. The primary design was a pre-post assessment of changes in depression severity. In addition, we collected blood samples at the same time points (pre- and post-intervention). All data were collected in 2012 at the Langley Porter Adult Psychiatry Clinic at the University of California, San Francisco. The blood samples were kept frozen while funding for the biomarker analysis was obtained. The university's institutional review board approved the study and all participants signed consents.

Participants were 8 female and 3 male outpatients, aged 20 to 48 years old, who met DSM-IV diagnoses of MDD through evaluation with the Structured Clinical Interview for DSM-IV diagnosis. This instrument and other ratings were completed by a clinical assessor not involved in the intervention. Participants were not taking medications for depression for at least 3 months, were not in psychotherapy, and had minimum Hamilton Depression Rating Score-17 items ( $\left.\mathrm{HAMD}_{17}\right)$ scores of 17 , indicating moderate to severe depressive symptoms upon 
entry into MBCT. Measures of depression severity and blood samples were collected pre - (T1) and post-MBCT intervention (T2). We used the $\mathrm{HAMD}_{17}$ as the primary outcome measure and also evaluated the change in inflammation using the CRP following MBCT. We utilized the Quick Inventory of Depressive Symptoms-Self Report-16 (QIDS-SR ${ }_{16}$ ) as a secondary outcome. We ruled out medical illness using a clinical review of systems. Participants were enrolled consecutively.

Depressed subjects participated in an 8-week MBCT structured group therapy intervention emphasizing mindfulness meditation training that included components of Cognitive-Behavioral Therapy. Classes were taught in 8 weekly consecutive sessions that were 2 hours and 15 minutes in duration and were led by a psychiatrist and a master's level therapist. Both the curriculum and format were developed by Segal et al., [13] with modifications by Eisendrath et al., [14] for an actively depressed population. Patients were asked not to start other treatments during the intervention.

Blood samples were collected within one week before T1 and after T2 and subjects were assessed by clinical history to rule out any concurrent or recent infections. To standardize potential diurnal CRP variation, whole blood samples were collected via venipuncture between 10 am and noon. The blood was then centrifuged @ 400rpm and the serum collected and stored at $-80^{\circ} \mathrm{C}$ pending analysis. All samples were shipped on dry ice for analysis at the same time to Myriad RBM for Human Inflammation ELISA bioassay for high-sensitivity CRP. The normal Myriad RBM range for CRP is 0.28 to $10 \mathrm{ug} / \mathrm{mL}$.

\section{Results and Discussion}

The cohort's clinical characteristics are described in table 1 . We note that the sample was predominantly female, similar to other depression studies. In addition, the sample was predominantly white, which may limit the generalizability of the findings. The sample had suffered depression for long periods and this may have motivated them to try a new approach to treatment so that this may also limit generalizability.

The mean change in CRP, HAMD ${ }_{17}$, and QIDS-SR 16 between $\mathrm{T} 1$ and $\mathrm{T} 2$ were analyzed using student's one-sample t-tests for significance. Mean CRP decreased from $1.82 \mathrm{mg} / \mathrm{L}$ at T1 to 1.32 at T2 $(\mathrm{t}(20)=2.21, \mathrm{p}=0.0517)$. This represented an effect size of 0.66 (Cohen's d, moderately large). The HAMD-17 score decreased significantly from 18.55 to 9.36 (t $(20)=5.76, \mathrm{p}<0.0001)$ with an effect size of 2.08 (Cohen's d, large). The QIDS-SR ${ }_{16}$ showed a similar decrease from 21.90 to $11.88(\mathrm{t}(18)=3.824, \mathrm{p}=0.001)$ with an effect size of 0.85 (Cohen's $d$, large). These outcomes are portrayed in table 2 .

To date, this is the first evidence that MBCT targeting depression has the potential to modulate inflammation in depressed patients without medical illness. CRP change from $\mathrm{T} 1$ to $\mathrm{T} 2$ was nearly significant $(\mathrm{p}=0.0517)$, and we expect that given a larger sample size, CRP change would have a better chance of meeting full significance. This finding raises many questions. If MBCT leads to decreased inflammation, how does it have this effect? Might change in inflammatory processes be the mediators of MBCT's effects on depression, or might MBCT reduce depression with consequent decreased inflammatory processes? Our small sample size limited our assessment of correlation between CRP and HAMD-17 scores and may be why this finding was statistically not significant. Given the growing evidence that a subset of depressed individuals are likely to

\begin{tabular}{|c|c|c|}
\hline Characteristics (n=11) & Mean/Description & $\begin{array}{c}\text { Standard } \\
\text { Deviation }\end{array}$ \\
\hline Patient age & 34.9 (20-48) years & 7.91 \\
\hline Education & 14.5 years & 2.5 \\
\hline Gender & 8F/3M & \\
\hline Ethnicity & 9 White/2 Hispanic & \\
\hline Employment Status & 4 Full/4 Part/3unemployed & \\
\hline Age of first major depressive episode & 24.8 & 6.64 \\
\hline Number of MDE & 3.4 & 1.51 \\
\hline Suicide Score (Item 3 HAMD) & 1 & 0.77 \\
\hline Length of MDE & 48.5 months & 53.7 \\
\hline $\begin{array}{c}\text { Per Cent free of psychotropic } \\
\text { medications>3 months }\end{array}$ & $100 \%$ & 8.54 \\
\hline BMI & 26.5 & \\
\hline No active substance abuse & 11 & \\
\hline \multicolumn{2}{|c|}{ Table 1: Clinical characteristics. } \\
\hline
\end{tabular}

\begin{tabular}{|c|c|c|c|}
\hline Measure Means & Pre-MBCT (T1) & Post-MBCT (T2) & Significance \\
\hline CRP & $1.82 \mathrm{ug} / \mathrm{mL}$ & $1.32 \mathrm{ug} / \mathrm{mL}$ & $\mathrm{p}=0.0517$ \\
\hline HAMD $_{17}$ & 18.55 & 9.36 & $\mathrm{p}<0.0001$ \\
\hline QIDS-SR $_{16}$ & 21.9 & 11.88 & $\mathrm{p}=0.001$ \\
\hline \multicolumn{4}{|c}{ Table 2: Depression and CRP outcomes. } \\
\hline
\end{tabular}

demonstrate increased inflammation [15,16], does inflammation level make an individual more or less sensitive to MBCT? Future research via a full, Randomized Controlled Trial (RCT) could address these questions as well as contribute to furthering the understanding of the pathophysiology of MDD.

CRP is an acute phase protein that responds to cytokines and gives evidence of inflammation. Cytokines have been shown to access the brain and cause negative effects on neurogenesis, neurotransmitter metabolism, and neuroendocrine function, all processes known to be involved in depression [7]. Research has shown that mindfulness meditation can also affect the brain through each of these domains [17-22]. How mindfulness meditation brings about its effects in these domains, and whether this involves inflammatory cytokines remains unclear. Kaliman et al., [23] found decreased pro-inflammatory gene (RIPK2 and COX2) expression in meditators compared with controls. Malarkey et al., [24] found that a mindfulness-based intervention for an employee program was associated with a decrease in CRP compared to controls that approached significance $(\mathrm{p}=0.08)$. If change in inflammation mediates MBCT effects on depression, it may be that MBCT affects inflammatory cytokines via similar mechanisms as delineated by Kaliman et al., [23] and Malarkey et al., [24].

\section{Conclusion}

This pilot study suggests that MBCT may modulate changes in $\mathrm{CRP}$ as reductions in depression severity occur. The study has several limitations. The sample was small, not randomized, and potentially affected by self-selection bias. Given the lack of a control group, changes in CRP or depression severity cannot be attributed with certainty to MBCT. Without a control group it is not possible to assess whether changes in depression may have mediated changes in CRP or vice versa. It is unknown if changes might have occurred naturally or 
due to other factors, such as the large age range of the sample. Without a randomized sample, we were also unable to evaluate the role of MBCT's putative mediators such as mindfulness meditation, enhanced self-compassion, decreased rumination, and perceived stress in decreasing inflammation. This investigation provides preliminary evidence for shaping more personalized and precise treatment by incorporating inflammatory marker information as a potential target of intervention, and it lays the groundwork for a more complete evaluation through a RCT. Such a trial would allow a formal mediational analysis to more clearly understand the mechanisms involved.

\section{Authors Contributions}

Stuart J Eisendrath, Molly James-Myers, Erin Gillung, Dhakshin Ramanatham designed and carried out the original data collection.

Kevin Delucchi performed the statistical analysis.

Owen Wolkowitz participated in the original design and interpretation of biomarker data.

Abby Hartzler and Walter Sipe participated in the drafting of the manuscript and its revisions. All authors read and approved the final manuscript.

\section{Acknowledgment}

The authors thank research assistants Lauren Erickson, MA, Chrystal Hogan, and the research participants.

\section{Funding}

This research was supported by a UCSF New Directions RAP grant. Dr. Eisendrath was supported by NCCAM/NIH grant R01AT004572-004.

\section{References}

1. Dowlati Y, Herrmann N, Swardfager W, Liu H, Sham L, et al. (2010) A meta-analysis of cytokines in major depression. Biol Psychiatry 67: 446-457.

2. Haroon E, Raison CL, Miller AH (2012) Psychoneuroimmunology meets neuropsychopharmacology: translational implications of the impact of inflammation on behavior. Neuropsychopharmacology 37: 137-162.

3. Maes M, Bosmans E, Suy E, Vandervorst C, DeJonckheere C, et al. (1991) Depression-related disturbances in mitogen-induced lymphocyte responses and interleukin-1 beta and soluble interleukin-2 receptor production. Acta Psychiatr Scand 84: 379-386.

4. Zorrilla EP, Luborsky L, McKay JR, Rosenthal R, Houldin A, et al. (2001) The relationship of depression and stressors to immunological assays: a meta-analytic review. Brain Behav Immun 15: 199-226.

5. Motivala SJ, Sarfatti A, Olmos L, Irwin MR (2005) Inflammatory markers and sleep disturbance in major depression. Psychosom Med 67: 187-194.

6. Hannestad J, DellaGioia N, Bloch M (2011) The effect of antidepressant medication treatment on serum levels of inflammatory cytokines: a meta-analysis. Neuropsychopharmacology 36: 2452-2459.

7. Miller AH, Maletic V, Raison CL (2009) Inflammation and its discontents: the role of cytokines in the pathophysiology of major depression. Biol Psychiatry 65: 732-741.
8. Chen HY, Cheng IC, Pan YJ, Chiu YL, Hsu SP, et al. (2011) Cognitive-behavioral therapy for sleep disturbance decreases inflammatory cytokines and oxidative stress in hemodialysis patients. Kidney Int 80: 415-422.

9. Thornton LM, Andersen BL, Schuler TA, Carson WE 3rd (2009) A psychological intervention reduces inflammatory markers by alleviating depressive symptoms: secondary analysis of a randomized controlled trial. Psychosom Med 71: 715-724.

10. Harley J, Luty S, Carter J, Mulder R, Joyce P (2010) Elevated C-reactive protein in depression: a predictor of good long-term outcome with antidepressants and poor outcome with psychotherapy. J Psychopharmacol 24: 625-626.

11. Dahl J, Ormstad H, Aass HC, Sandvik L, Malt UF, et al. (2016) Recovery from major depressive disorder episode after non-pharmacological treatment is associated with normalized cytokine levels. Acta Psychiatr Scand .

12. Krogh J, Benros ME, Jørgensen MB, Vesterager L, Elfving B, et al. (2014) The association between depressive symptoms, cognitive function, and inflammation in major depression. Brain Behav Immun 35: 70-76.

13. Segal ZV, Williams JM, Teasdale JD (2002) Mindfulness-based cognitive therapy for depression: A new approach to preventing relapse. Guilford Press, New York, USA.

14. Eisendrath SJ, Delucchi K, Bitner R, Fenimore P, Smit M, et al. (2008) Mindfulness-based cognitive therapy for treatment-resistant depression: a pilot study. Psychother Psychosom 77: 319-320.

15. Halder I, Marsland AL, Cheong J, Muldoon MF, Ferrell RE, et al. (2010) Polymorphisms in the CRP gene moderate an association between depressive symptoms and circulating levels of C-reactive protein. Brain Behav Immun 24: $160-167$

16. Raison CL, Miller AH (2011) Is depression an inflammatory disorder? Curr Psychiatry Rep 13: 467-475.

17. Chiesa A, Serretti A (2010) A systematic review of neurobiological and clinical features of mindfulness meditations. Psychol Med 40: 1239-1252.

18. Davidson RJ, Kabat-Zinn J, Schumacher J, Rosenkranz M, Muller D, et al. (2003) Alterations in brain and immune function produced by mindfulness meditation. Psychosom Med 65: 564-570.

19. Fang CY, Reibel DK, Longacre ML, Rosenzweig S, Campbell DE, et al. (2010) Enhanced psychosocial well-being following participation in a mindfulness-based stress reduction program is associated with increased natural killer cell activity. J Altern Complement Med 16: 531-538.

20. Pace TW, Negi LT, Adame DD, Cole SP, Sivilli TI, et al. (2009) Effect of compassion meditation on neuroendocrine, innate immune and behavioral responses to psychosocial stress. Psychoneuroendocrinology 34: 87-98.

21. Rubia K (2009) The neurobiology of Meditation and its clinical effectiveness in psychiatric disorders. Biol Psychol 82: 1-11.

22. Witek-Janusek L, Albuquerque K, Chroniak KR, Chroniak C, Durazo-Arvizu $R$, et al. (2008) Effect of mindfulness based stress reduction on immune function, quality of life and coping in women newly diagnosed with early stage breast cancer. Brain Behav Immun 22: 969-981.

23. Kaliman $P$, Alvarez-López MJ, Cosín-Tomás M, Rosenkranz MA, Lutz A, et al. (2014) Rapid changes in histone deacetylases and inflammatory gene expression in expert meditators. Psychoneuroendocrinology 40: 96-107.

24. Malarkey WB, Jarjoura D, Klatt M (2013) Workplace based mindfulness practice and inflammation: a randomized trial. Brain Behav Immun 27: 145-154. 